\title{
to Convey Emotion
}

\section{Norah Jean-Charles and Leonard Hayes Department of Computer Science}

Advisor: Dr. Paola Spoletini

\section{ABSTRACT}

- The response of the interviewee, such as his or her emotional state, plays an important role on the quality of the collected requirements and helps the interviewer respond accordingly.

- Creating a web app allows interviewers to see the statistics gathered from the E4 wristband and the emotion that the interviewee is experiencing in real-time.

- Biofeedback information is being extracted from the E4 wristband using a serverto-client console method and supervised machine learning techniques are being used to display emotion.

\section{BACKGROUND}

- Requirements elicitation interviews are the most commonly used technique to gather requirements. This is a crucial and difficult activity on which the quality of software depends on.

- Emotions such as stress help determine whether or not an interview is successful or not by dictating the level of attention and engagement of the interviewee. Emotions are not always easy to identify by observation. However, they can be detected by looking at various vitals (e.g. rate of blood flow, heart rate, and temperature) and voice parameters.

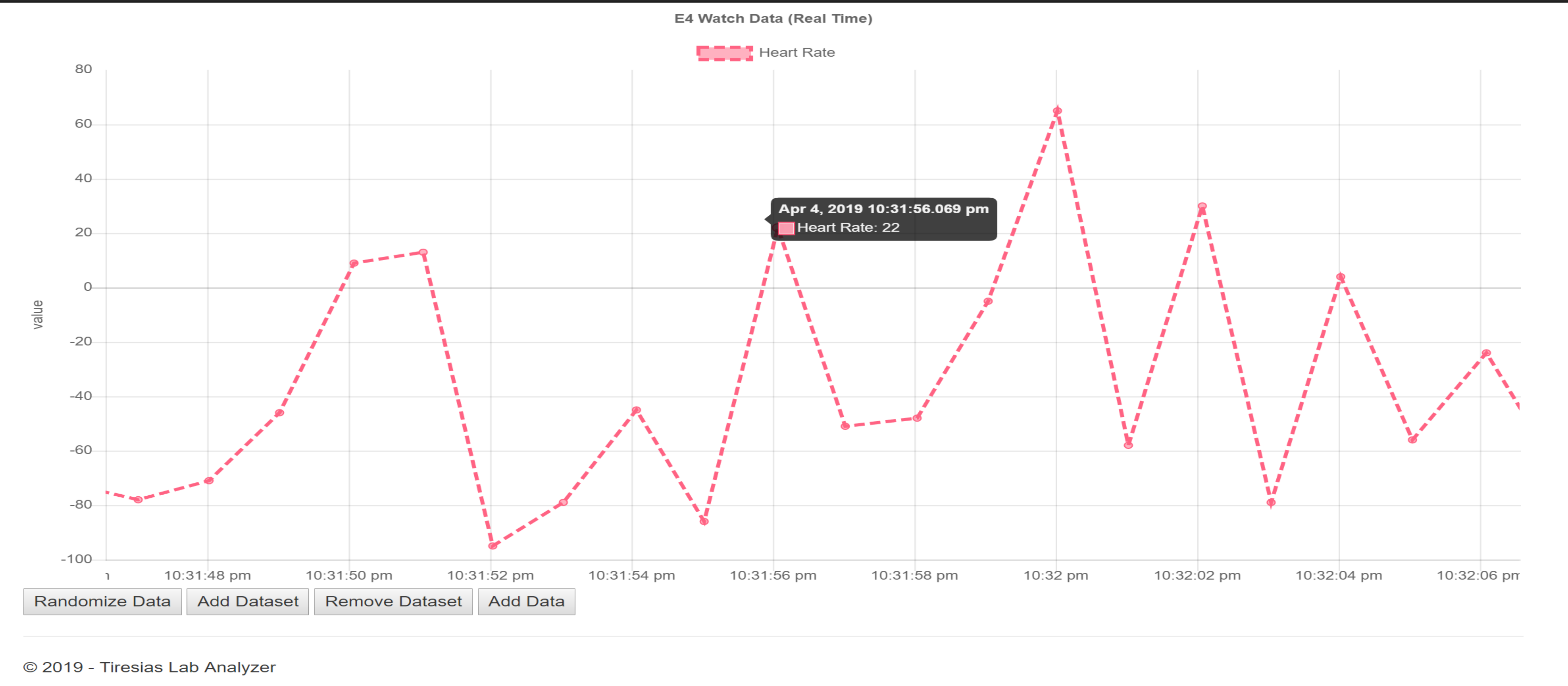

Web application giving E4 wristband data in real-time

\section{METHODS}

Web Application:

- Use ASP.NET core framework to build app

- Use server-to-client console method

- Program with C\#, Razor, MVC, HTML, \& JS

- Use of Chart.js package to display data from watch

Machine Learning:

- Retrieve and clean biofeedback data from the E4 wristband

- Collect and enter data from image surveys

- Use logistic regression to average the heart beats with the other biofeedback data

- Build ANN using Python and Tensorflow

\section{Result of Python script consolidating biofeedback data from different files}

\section{CONCLUSION}

The intended outcome of our on-going project is to have a complete web application that provides the interviewer with a GUI interface for collecting and analyzing data on the interviewee, as well as conveying the emotion of the interviewer using machine learning techniques.

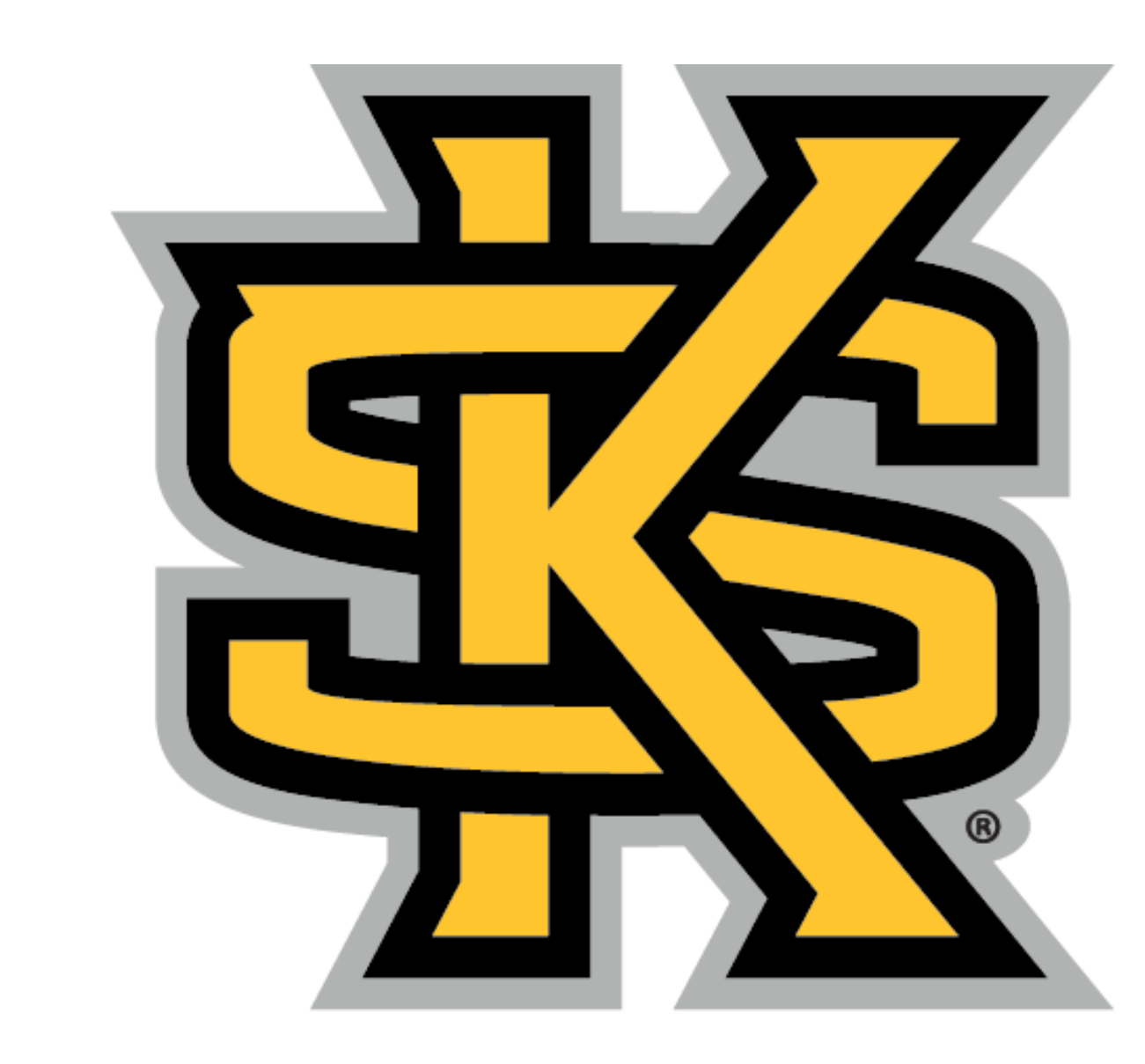

KENNESAW STATE

U N I V E R S T Y
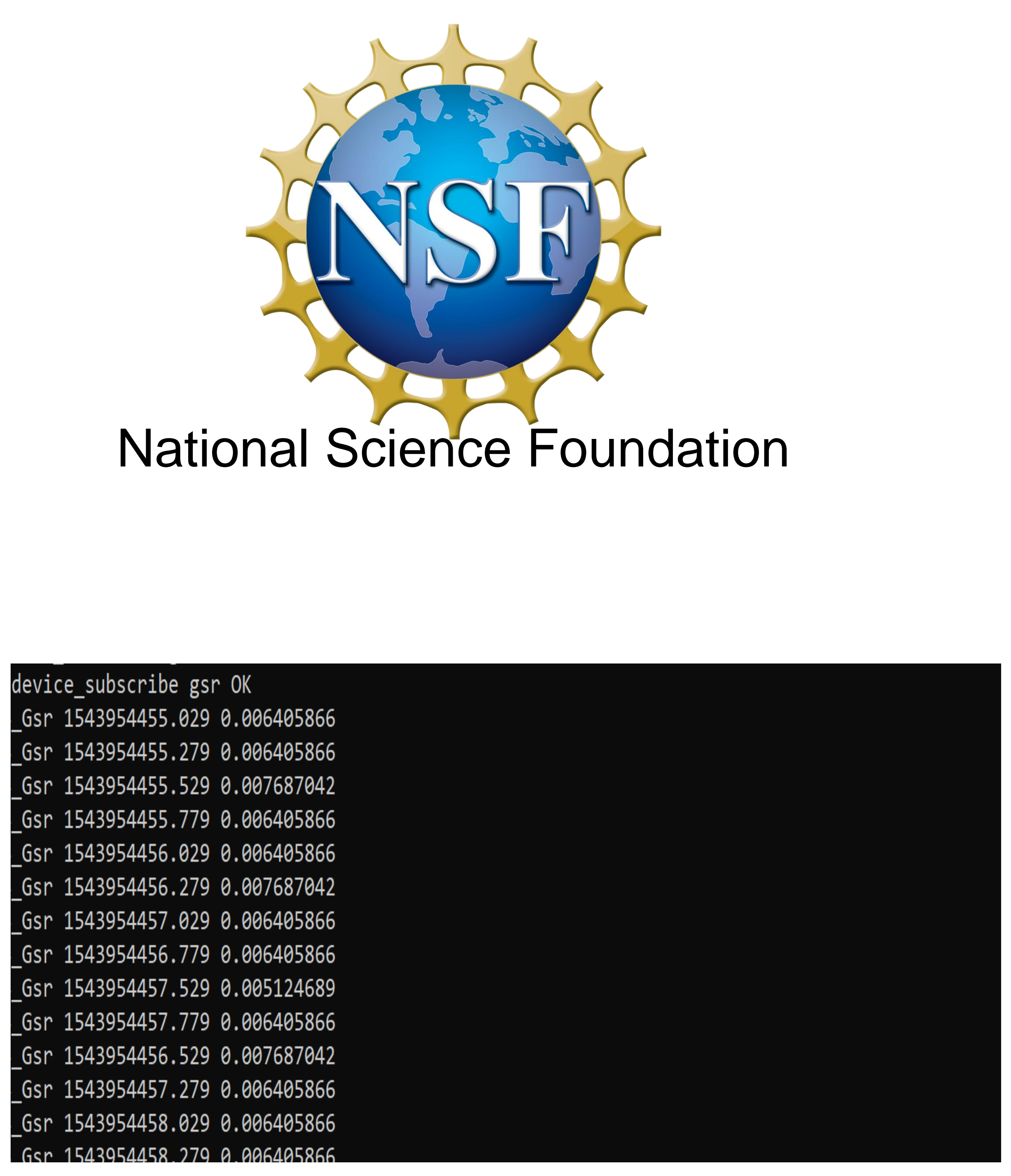

Empatica server-to-client console method giving Gsr values

\section{ACKNOWLEDGMENTS}

This project is funded by NSF under grant CCF-1718377. Any opinions, findings, and conclusions or recommendations expressed in this material are those of author(s) and do not necessarily reflect the views of the National Science Foundation. We would like to thank Alex Drennan and Gregor Haas, computer science students, who have been instrumental in the development of the ANN for conveying emotion. We would also like to thank Dr. Mohammed Aledhari, Assistant Professor in Computer Science at KSU, for his advice on machine learning techniques. 